WE3F-16

\title{
COMPARATIVE PERFORMANCE OF THREE DIFFERENT CPW BANDPASS FILTERS
}

\author{
Fang-Lih Lin and Ruey-Beei Wu \\ Department of Electrical Engineering, Rm. 340 \\ National Taiwan University, Taipei, Taiwan 10617, ROC
}

\begin{abstract}
This paper addresses the performance comparison between three kinds of coplanar waveguide bandpass filter designs: ribbon-of-brickwall, impedance transformer type, and gapcoupled filters. Several analysis models have been employed to investigate the high frequency characteristics of the three kinds of filters including the scattering parameters and the power loss. The numerical results are in good agreement with the measured data.
\end{abstract}

\section{INTRODUCTION}

Filter is one of the most important passive components in the microwave circuits. In the past, most of the filters are realized in microstrip lines for planar circuit applications. As the CPW experiences a growing demand in the MIC and MMIC circuits, several CPW filter designs have been found in the literature [1]-[4]. In those works, the CPW discontinuities appearing in the filter structures are characterized as lumped circuits using the quasi-static approximation. This approach requires little computational effort and can accurately predict the filter performance, such as center frequency, bandwidth, etc. However, the quasi-static model can not account for electromagnetic radiation, coupling, and surface wave excitation, which becomes significant with increasing frequency. Consequently, the full-wave analysis is required for characterizing the high frequency behaviors of CPW filters. In this paper, three kinds of coplanar waveguide bandpass filters - ribbon-ofbrick-wall (RBW), impedance transformer type,

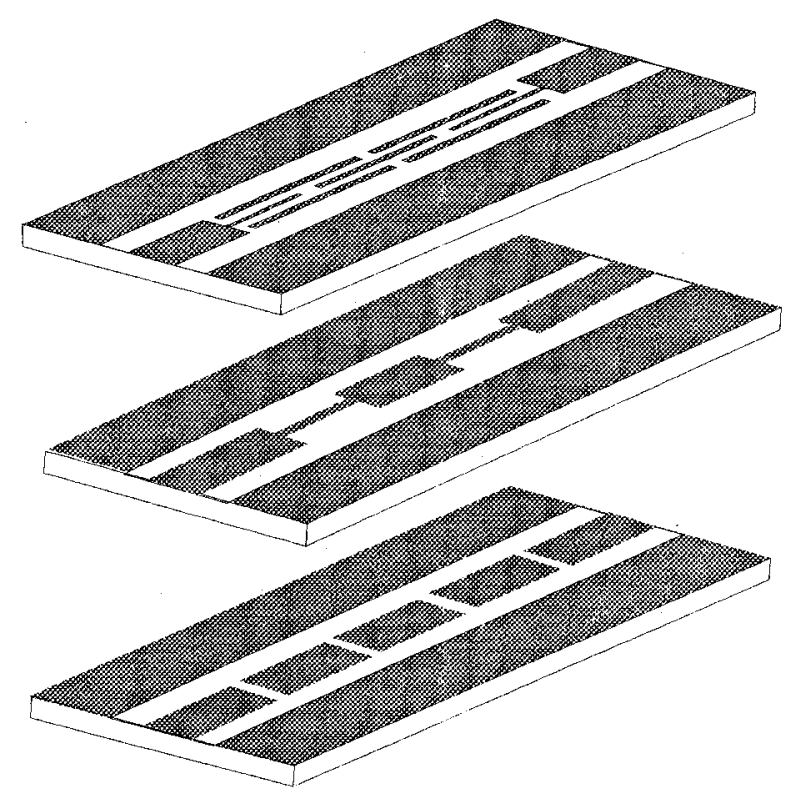

Fig.1: Layout pattern of three coplanar waveguide band-pass filters: ribbon-of-brickwall type, impedance transformer type, and gap-coupled type.

and gap-coupled filters - are designed and realized. Comparative studies for the full-wave characteristics of these three kinds of filters are performed using several analysis models, including the recently developed matrix-penciled moment method analysis [5].

\section{FILTER DESIGN}

Fig.1 shows the basic layout patterns of the bandpass filters implemented for CPW structures. The building element of the RBW filter is a 


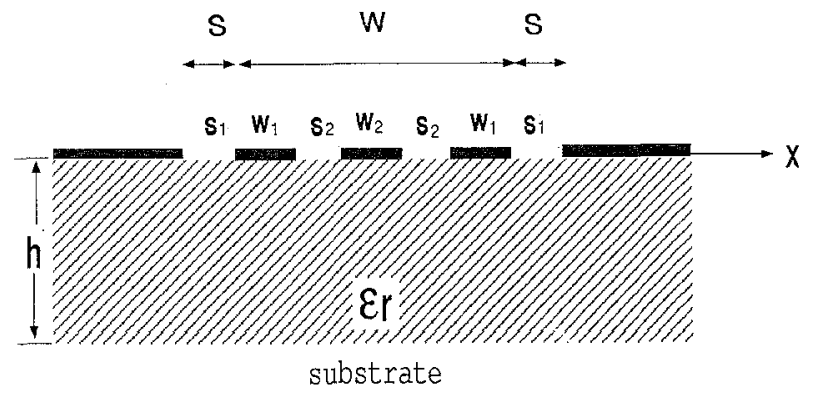

Fig.2: The definition of geometric parameters used in Tables 1, 2, and 3

quarter-wavelength CPW open-end series stub. Detailed design concept and steps can be found in [4]. The design of impedance transformer type filter is based on the quarter-wave transformers. Detailed design procedure can be found in [6]. The gap-coupled filter consists of several half-wave resonators which are separated by gaps of different widths to provide suitable capacitive coupling. Detailed design procedure can be found in [7].

Tables 1, 2, and 3 list the values of the geometric parameters for these three kinds of filters to be designed for several different bandwidth specifications. The definitions of geometric parameters are shown in Fig.2. For the case of RBW filter with $3 \%$ bandwidth, it is impossible to find suitable geometric parameters from the design chart for realizing the coupling parameter K. Even for 10 $\%$ bandwidth case, the slot width $S_{1}$ is only 0.01 $\mathrm{mm}$, about $\frac{1}{93}$ of the central signal strip width $W_{2}$. This causes a great concern in the fabrication and reliability. It is thus concluded that the filters of RBW type are more suitable for wider bandwidth applications.

From Table 2, it can be found that the ratio of $Z_{1}$ to $Z_{2}$ increases significantly as the bandwidth decreases for impedance transformer type filter. This is undesired for practical implementation. For example, consider a bandpass filter design of $3 \%$ bandwidth on a CPW system having characteristic impedance of $100 \Omega$. The value of $Z_{1}$ and $Z_{2}$ will be $1280 \Omega$ and $5 \Omega$, which are impossible to realize. Therefore, the impedance transformer type filters are more suitable for wider bandwidth applications.

For gap-coupled filter, if filters of wider bandwidths are desired, larger coupling capacitances are required. In those cases, the gap width may become too small to be implemented. Therefore, the gap-coupled filters are nore suitable for narrow bandwidth applications.

Table 1

\begin{tabular}{|c|c|c|c|c|c|c|}
\hline BW & section & K & S1 & S2 & W1 & W2 \\
\hline $3 \%$ & 1,4 & 0.167 & 0.021 & 0.301 & 0.048 & 0.902 \\
\hline & 2,3 & 0.036 & - & - & - & - \\
\hline $10 \%$ & 1,4 & 0.286 & 0.079 & 0.282 & 0.096 & 0.845 \\
\hline & 2,3 & 0.117 & 0.011 & 0.310 & 0.024 & 0.931 \\
\hline $20 \%$ & 1,4 & 0.371 & 0.267 & 0.306 & 0.036 & 0.917 \\
\hline & 2,3 & 0.225 & 0.042 & 0.291 & 0.072 & 0.874 \\
\hline $40 \%$ & 1,4 & 0.450 & 0.695 & 0.498 & 0.088 & 0.427 \\
\hline & 2,3 & 0.387 & 0.239 & 0.431 & 0.184 & 0.370 \\
\hline $60 \%$ & $1-4$ & 0.579 & 0.950 & 0.320 & 0.320 & 0.320 \\
\hline
\end{tabular}

Table.1: The electrical and geometric parameters for RBW filters with different bandwidths. Order of filters is 3 and the center frequency is at $10 \mathrm{GHz}$. The characteristic impedance of feed line is $100 \Omega$. The width of signal strip is $1.6 \mathrm{~mm}$ and slot width is $0.95 \mathrm{~mm}$. The thickness of substrate is $1.58 \mathrm{~mm}$ and dielectric constant is 2.2 .

Table 2

\begin{tabular}{|c|c|c|c|c|c|}
\hline $\mathrm{BW}$ & $z_{1} / z_{0}$ & $z_{2} / z_{0}$ & $z_{1} / z_{2}$ & $\sec 1 \mathrm{w} / \mathrm{s}$ & $\sec 2 \mathrm{w} / \mathrm{s}$ \\
\hline $3 \%$ & 12.8 & 0.05 & 258 & - & - \\
\hline $10 \%$ & 10.4 & 0.15 & 69.5 & - & - \\
\hline $20 \%$ & 5.17 & 0.30 & 17.2 & - & - \\
\hline $40 \%$ & 2.25 & 0.65 & 3.46 & $0.16 / 1.67$ & $2.84 / 0.33$ \\
\hline $60 \%$ & 1.80 & 1.07 & 1.68 & $0.33 / 1.59$ & $1.45 / 1.03$ \\
\hline
\end{tabular}

Table.2: The electrical and geometric parameters for impedance transformer type filters with different bandwidths. Other specifications are same with Table 1.

Table 3

\begin{tabular}{|c|c|c|c|c|}
\hline BW & $\mathrm{C} 1,4(\mathrm{fF})$ & $\mathrm{C} 2,3(\mathrm{fF})$ & gap1,4 & gap2,3 \\
\hline $3 \%$ & 28.22 & 5.68 & 0.254 & 1.57 \\
\hline $10 \%$ & 55.45 & 19.19 & 0.008 & 0.40 \\
\hline $20 \%$ & 88.04 & 40.11 & 0.002 & 0.05 \\
\hline $40 \%$ & 164.86 & 97.74 & - & 0.0001 \\
\hline $60 \%$ & 299.04 & 230.50 & - & - \\
\hline
\end{tabular}

Table.3 : The electrical and geometric parameters for gap-coupled filters with different bandwidths. The width of signal strip is $1.0 \mathrm{~mm}$ and slot width is $0.57 \mathrm{~mm}$. Other specifications are same with Table 1 . 


\section{NUMERICAL RESULTS}

Three different approaches are employed to characterize the performance of filters. For quasistatic analysis, equivalent lumped circuit models of discontinuities are evaluated [8] [9]. Combining the lumped circuit model for the discontinuities and the transmission line model for the CPW sections yields the equivalent circuits of the whole filter. The scattering parameters can then be obtained via common circuit simulators, e.g., SPICE or Touchstone.

For S-matrix method, the higher order modes on the CPW lines as well as the coupling between different sections are neglected. The S-matrix of each constitutive section of filter are first extracted by full-wave analysis [5], then the whole filter structures are characterized by cascading these Smatrices. In addition, a full-wave algorithm is employed to fully account for all high frequency effects, which hybridizes moment method solution for the mixed potential integral equation and the matrix pencil approach for scattering parameters. The whole filter structure is analyzed altogether to obtain directly the final scattering parameters of the filters.

Fig.3 show the simulation results by three approaches and measured data for the $S_{21}$ of these three kinds of filters. Also shown in the figure for comparison are the experimental data measured under the TRL calibration and good agreement can be found among them. For impedance transformer type filter, it can be found from Fig.3(b) that the attenuation is not satisfactory in the outof-band region due to the rapid repeated appearance of adjacent pass bands. Although this can be improved by increasing the filter's order, the resultant increase in total size plagues the applications of this kind of filters.

The power losses including the radiation and surface wave losses of the three filters are shown in Fig.4. Among the three approaches, only the full-structure analysis is applicable for such analysis. It is found that the power losses of RBW and impedance transformer type filters are considerably smaller in the pass-band region than that of the gap-coupled filter. The large power loss for the gap-coupled filter depicts that significant radiation is resulted from the gaps between resonators. The large power loss should be taken into consideration in designing the gap-coupled bandpass filters for narrow bandwidth specifications.
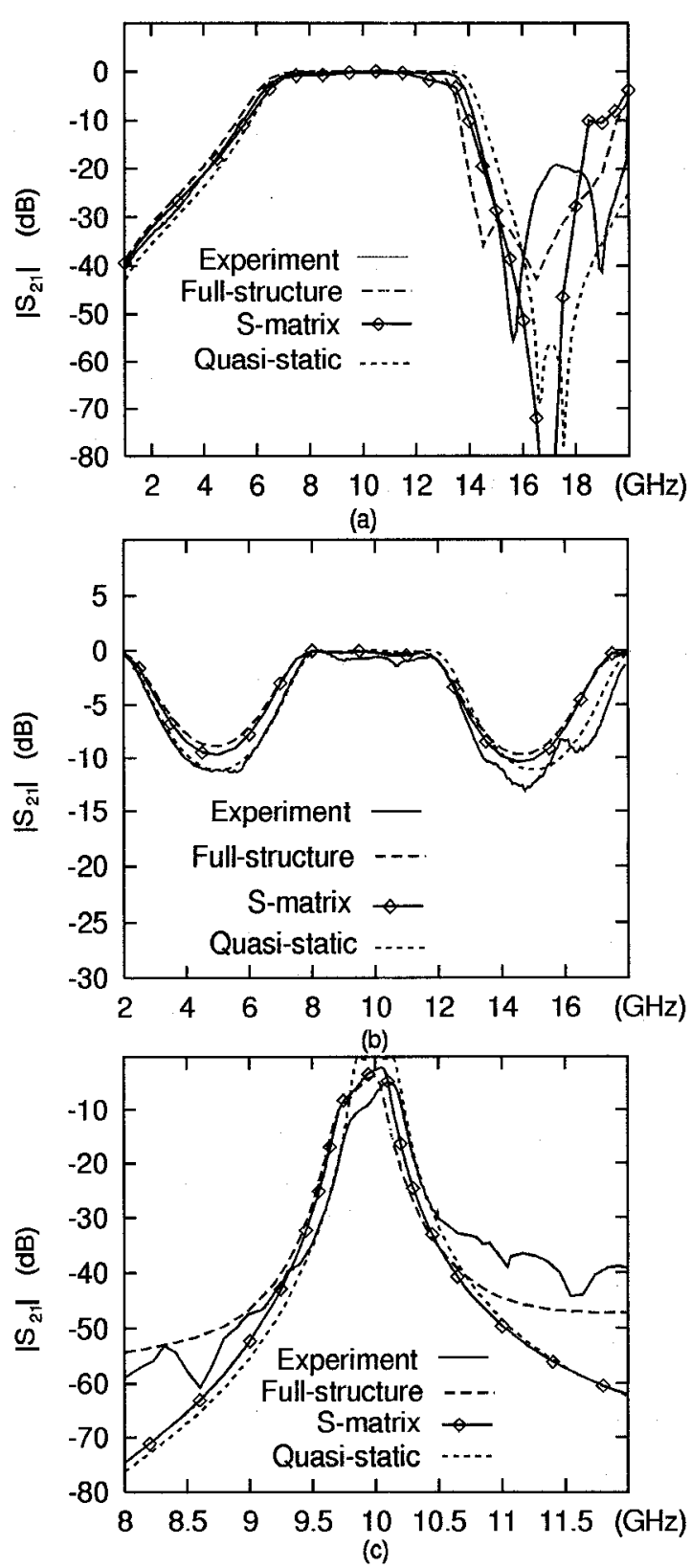

Fig.3 : Simulated and measured scattering parameters for (a) periodic RBW filter, (b) impedance transformer type filter, and (c) gap-coupled filter. The characteristic impedances of feeding and output CPW lines are $100 \Omega$. The center frequencies are at $10 \mathrm{GHz}$ and the bandwidths are $60 \%$, $40 \%$, and $3 \%$ for RBW, impedance transformer type, and gap-coupled filters, respectively. 


\section{CONCLUSIONS}

As demonstrated above, it is found that both RBW and impedance transformer type filters can achieve wider bandwidth with low loss, but the former has better out-of-band rejection behavior. The gap-coupled filter can attain narrower bandwidth and good stop-band characteristics, with a large pass-band loss due to the radiation loss from the gaps. The proper type of filters should be chosen in the design according to different bandwidth requirements.

The three kinds of bandpass filters presented in this paper can yield good filter performance. They are all uni-planar $\sim$ requiring only one metallization and no air-bridge. Also, they are compact in size and suitable for MIC and MMIC applications.

\section{References}

[1] D. F.-Williams and S. E. Schwarz, "Design and performance of coplanar waveguide bandpass filters," IEEE Trans. Microwave Theory Tech., vol.MTT-31, pp.558-566, July 1983.

[2] C. Nguyen, "Broadside-coupled coplanar waveguide and their end-coupled band-pass filter applications," IEEE Trans. Microwave Theory Tech., vol.MTT-40, pp.2181-2189, Dec. 1992.

[3] J. K. A. Everard and K. K. M. Cheng "High performance direct coupled bandpass filters on coplanar waveguide," IEEE Trans. Microwave Theory Tech., vol.MTT-41, pp.15681573, Sept. 1993.

[4] F. L. Lin, C. W. Chiu, and R. B. Wu, "Coplanar waveguide bandpass filter- a ribbon-ofbrick-wall design," IEEE Trans. Microwave Theory Tech. pp.1589-1596, July 1995.

[5] M. D. Wu, S. M. Deng, R. B, Wu, and P. Hsu, "Full-wave characterization of mode conversion in a coplanar waveguide right-angled bend," IEEE Trans. Microwave Theory Tech. vol.MTT-43, pp.2532-2538, Nov. 1995.

[6] R. E. Collin, Foundations for Microwave Engineering., 2nd ed., New York: McGraw Hill, 1992 , ch. 8.

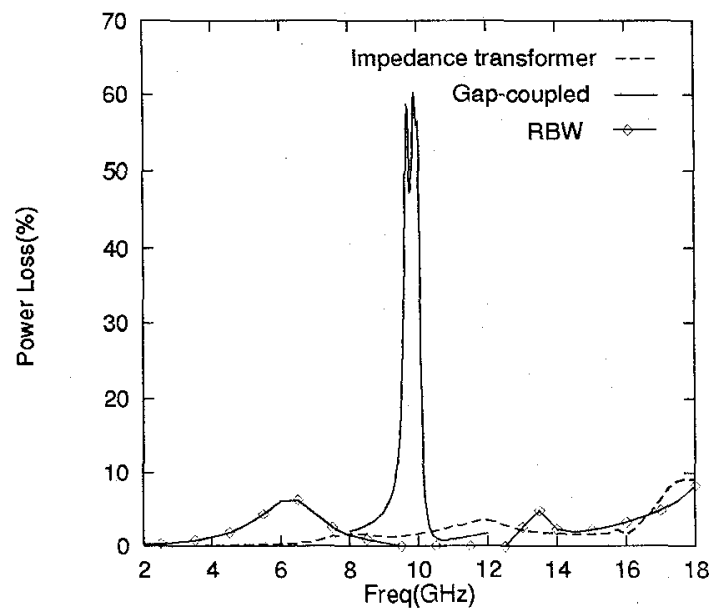

Fig.4: Power losses calculated by the fullstructure analysis for the three different kinds of filters considered in Fig. 3.

[7] G. L. Matthaei, L. Young, and E. M. T. Jones, Microwave Filters, Impedance-Matching Network, and Coupling Structures. Dedham, Massachusetts: Artech House, 1980.

[8] M. H. Mao, R. B. Wu, C. H. Chen, and C. H. Lin, "Characterization of coplanar waveguide open end capacitance - theory and experiment," IEEE Trans. Microwave Theory Tech. vol.MTT-42, pp.1016-1024, June 1994.

[9] C. W. Chiu and R. B. Wu, "A moment method analysis for coplanar waveguide discontinuity inductances," IEEE Trans. Microwave Theory Tech. vol. MTT-41, pp.15111514, Sept. 1993. 